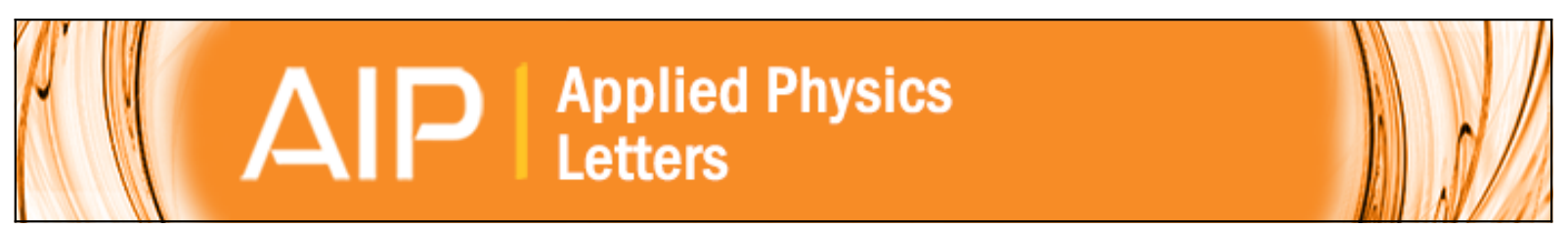

Polarization screening in polymer ferroelectric films: Uncommon bulk mechanism

E. Mikheev, I. Stolichnov, A. K. Tagantsev, and N. Setter

Citation: Applied Physics Letters 101, 132905 (2012); doi: 10.1063/1.4754146

View online: http://dx.doi.org/10.1063/1.4754146

View Table of Contents: http://scitation.aip.org/content/aip/journal/apl/101/13?ver=pdfcov

Published by the AIP Publishing

Over 700 papers \& presentations on

multiphysics simulation vew now

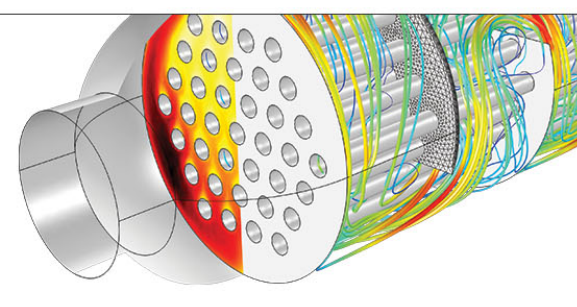




\title{
Polarization screening in polymer ferroelectric films: Uncommon bulk mechanism
}

\author{
E. Mikheev, I. Stolichnov, A. K. Tagantsev, and N. Setter \\ Ceramics Laboratory, EPFL-Swiss Federal Institute of Technology, Lausanne 1015, Switzerland
}

(Received 21 June 2012; accepted 6 September 2012; published online 27 September 2012)

\begin{abstract}
Charge compensation at the interface is a fundamental phenomenon determining the operation conditions of thin-film devices incorporating ferroelectrics. The underlying mechanisms have been thoroughly addressed in perovskite ferroelectrics where the charge compensation originates from injection through the interface-adjacent layer. Here, we demonstrate that polarization screening in the polymer ferroelectric polyvinylidene fluoride-trifluoroethylene $(\mathrm{P}(\mathrm{VDF}-\mathrm{TrFE}))$ films can be dominated by charge injection through the bulk, unlike ferroelectric oxides. The experimental evidence relies on polarization imprint under applied field and time-dependence of the dielectric constant. A linearized electrostatic model correctly accounts for the observed trends and links their occurrence to the unique properties of P(VDF-TrFE). (C) 2012 American Institute of Physics. [http://dx.doi.org/10.1063/1.4754146]
\end{abstract}

Polyvinylidene fluoride-trifluoroethylene or P(VDF$\mathrm{TrFE})$ is a unique co-polymer system exhibiting robust ferroelectricity in an organic compound. ${ }^{1-3}$ It attracts interest for fully organic and hybrid organic/inorganic non-volatile memories, ${ }^{4-7}$ due to an uncommon blend of properties: relatively high spontaneous polarization combined with excellent stability and the possibility of low-temperature deposition compatible with virtually any substrate. In parallel, a number of exciting advanced applications of P(VDFTrFE) have emerged in recent years, such as ferroelectric control of ferromagnetism in dilute magnetic semiconductors $^{8}$ and enhancement of organic solar cell efficiency. ${ }^{9}$

Given the technological interest in the heterostructures incorporating PVDF-TrFE layers, the properties of the interfaces between the ferroelectric polymer and adjacent metal or semiconductor are of high interest. Polarization charge compensation is a phenomenon of particular importance as it determines the net polarization governing properties of the functional interfaces. The mechanisms of polarization compensation have been previously investigated in traditional perovskite ferroelectrics in relationship with the practical issue of polarization imprint. ${ }^{10,11}$ Here, we show that in P(VDF-TrFE) the physics of polarization screening is radically different from the well-established picture in ferroelectric oxide thin films.

The screening phenomenon is associated with accumulation of the compensating free charges on the polar faces of the ferroelectric crystal, impeding polarization reversal. This yields a logarithmic dependence of coercive voltage $V_{C}$ on time after poling $t_{\text {impr }}: V_{C} \sim \log \left(t_{\text {impr }}\right)$. It is commonly described in terms of charge injection through an electrodeadjacent dielectric or "dead" layer, driven by the large electric field perceived by this layer. ${ }^{10,11}$ The essential feature associated with this mechanism is the behavior under applied electric bias parallel to the polarization of the sample, which was shown to accelerate the imprint process ${ }^{10}$ by acting in combination with the field of the depolarization origin. Here, we show that in $\mathrm{P}(\mathrm{VDF}-\mathrm{TrFE})$, applied bias of this orientation has a reverse effect (slowing the imprint), indicating that the path of compensating charges is opposite in its direction and associated with the electric field in the ferroelectric bulk.
Experimental investigations were conducted on $\mathrm{Au} /$ $\mathrm{P}(\mathrm{VDF}-\mathrm{TrFE}) / \mathrm{Au}$ capacitors. The 300-nm thick ferroelectric polymer was spun on the bottom $\mathrm{Au}$ from a solution with a 77/23\% VDF/TrFE composition. The 100-nm top Au layer was patterned by photolithography into electrodes of 0.2 $\mathrm{mm}^{2}$ area. Our experimental study of imprint is illustrated in Fig. 1(a): it is initiated by a measurement of ferroelectric hysteresis under a $1 \mathrm{kHz}$ triangular voltage $(V)$ pulse, setting the ferroelectric polarization $P_{f}$ to positive. Then the voltage cycling is repeated, noting the increase of coercive voltage as a function of total imprint time $t_{\text {impr }}$ after initial poling. The history of the sample was erased with a sequence of 30 pulses. The experiment was repeated with 5 and $10 \mathrm{~V}$ bias applied in between hysteresis cycles, with polarity aligned with the ferroelectric polarization.

Figure 1(b) shows the resulting imprint effect as seen directly in the polarization hysteresis. Applied bias with the polarity maintaining the poled state clearly slows down the increase of $V_{C}$, which is shown as a function of $t_{\text {impr }}$ in Fig. 1(c). This direction of the bias effect is incompatible with the classical situation of imprint driven by the field in the interface-adjacent dielectric layer. On the other hand, a scenario of imprint driven by depolarization field in the ferroelectric bulk is consistent with our experimental observations.

The phenomenon of polarization screening in the studied $\mathrm{Au} / \mathrm{P}(\mathrm{VDF}-\mathrm{TrFE}) / \mathrm{Au}$ capacitors is illustrated in Fig. 2. $\mathrm{P}(\mathrm{VDF}-\mathrm{TrFE})$ is a semi-crystalline polymer with a majority crystalline ferroelectric phase and a minority amorphous dielectric phase (Fig. 2(a)). A qualitative description of such a system can be obtained using a one-dimensional multilayer model (Fig. 2(b)), which in turn is electrostatically equivalent to a model sandwich of a ferroelectric layer of thickness $h$ and a thin near-electrode dielectric layer of thickness $d$ (Fig. 2(c)). The difference between the sandwich and the system in Fig. 2(a) is basically an additional shunt connected capacitor which is of a secondary importance for the imprint phenomenon. Thus, we adopt the sandwich model for the discussion of our experimental data. In the context of ferroelectric oxides, this model successfully describes imprint as a result of charge transport across the dielectric layer, with 

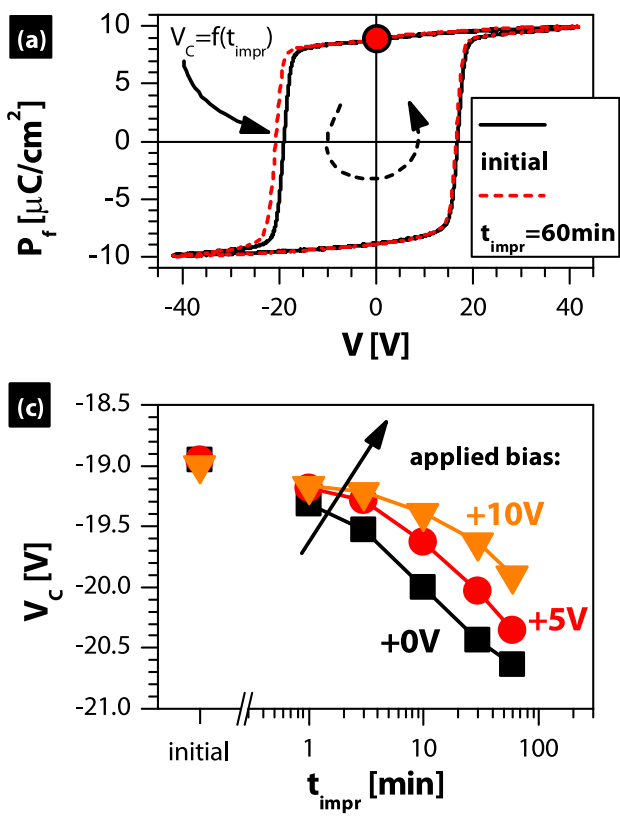

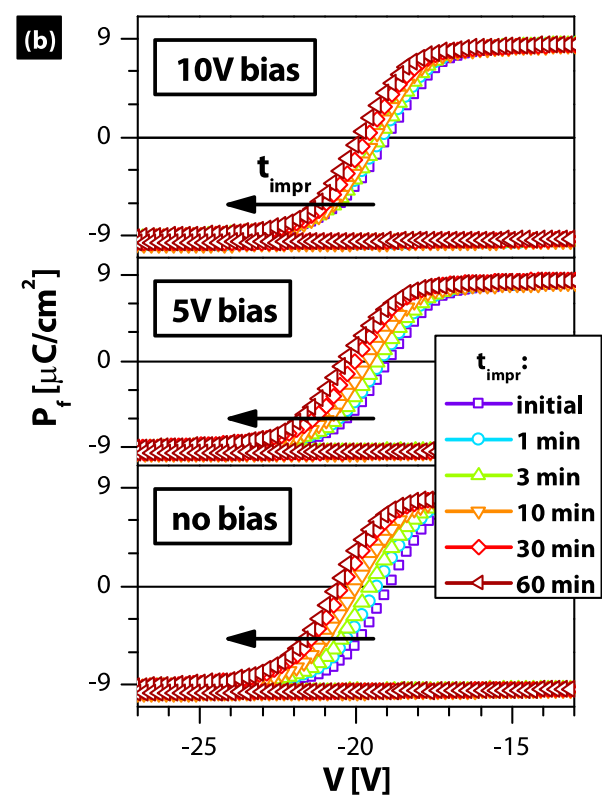

FIG. 1. (a) Ferroelectric hysteresis of $\mathrm{P}(\mathrm{VDF}-\mathrm{TrFE})$. The measurement of imprint in the positive polarization state (red dot) was carried out by repeating the measurement at various stages of the process. Hysteresis at $t_{\text {impr }}=0$ and $60 \mathrm{~min}$ are shown. (b) Imprint effect as seen in the ensemble of ferroelectric hysteresis data. Positive bias of 0,5 , and $10 \mathrm{~V}$ (along the polarization) was maintained in between hysteresis measurements. (c) Summary of imprint effect on coercive field at variable bias, which slows down the process. subsequent charge accumulation at the ferroelectric/dielectric interface.

In the model accounting for the imprint in ferroelectric oxide films, the driving force for this transport is a very high electric field $E_{d} \approx P_{f} / \kappa_{d} \varepsilon_{0}$ perceived by this dielectric layer. ${ }^{11}$ (Here, $P_{f}$ is the ferroelectric polarization and $\kappa_{d}$ the dielectric constant of the non-ferroelectric layer.) If an additional bias $U$ is applied along $P_{f}$ and $E_{d}$, the charge transport across the dielectric layer is enhanced, accelerating the imprint process. The trend of imprint slowing down under such bias (Fig. 1(b)) indicates that this process in $\mathrm{P}(\mathrm{VDF}-\mathrm{TrFE})$ cannot result from $E_{d}$. Instead, we suggest that it is driven by $E_{f}$, the depolarizing electric field perceived by the ferroelectric bulk, which is "negative," e.g., oriented opposite to $P_{f}$ and $E_{d}$.

As a further support of this argument, we present experimental data on the time-variation of the dielectric response, which is an additional manifestation of polarization compensation in ferroelectric thin films. ${ }^{12}$ Figure 3(a) illustrates that the dielectric susceptibility $\chi_{f}$ of P(VDF-TrFE) exhibits a well-defined logarithmic dependence on time after poling

$$
\chi_{f}=\chi_{f}(0)-\nu \cdot \ln \left(1+t / \tau_{0}\right),
$$

where $\chi_{f}(0), \nu$, and $\tau_{0}$ are, respectively, the initial value of $\chi_{f}$, the degradation rate, and a time constant. This law is obeyed
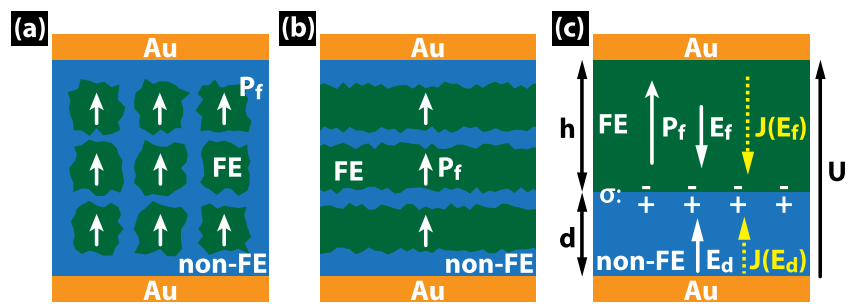

FIG. 2. Illustration of polarization compensation in an $\mathrm{Au} / \mathrm{P}(\mathrm{VDF}-\mathrm{TrFE}) / \mathrm{Au}$ capacitor. (a) $\mathrm{P}(\mathrm{VDF}-\mathrm{TrFE})$ is a semicrystalline polymer with a coexistence of crystalline ferroelectric (FE) and amorphous dielectric (non-FE) phases. (b) Reduction of the system to a one-dimensional multilayer. (c) The equivalent double-layer sandwich model. over a large span of low-frequency dielectric response and over many decades of time after polarization switching. The general trend of dielectric susceptibility decrease reflects the time-dependent polarization compensation and the depolarization field decay. The most intriguing aspect of this behavior is its dependence on electrical bias applied after the poling pulse, shown in Fig. 3(b). The speed of the degradation process, described by the $\nu$ parameter, is slowed by bias oriented along $P_{f}$ (Fig. 3(c)), implying a slower compensation of the polarization. Following analysis further clarifies this point.

In our approach, we show first that the depolarizing electric field $E_{f}$ follows a logarithmic dependence similar to Eq. (1), assuming that the polarization screening is driven by the charge transport across the ferroelectric layer. The time dependence of $E_{f}$ can be obtained from the system of equations describing the potential drop of voltage $U$ across the sandwich and the continuity of electric displacement

$$
\begin{gathered}
U=d E_{d}+h E_{f}, \\
P_{f}+\varepsilon_{0} \kappa_{b} E_{f}-\varepsilon_{0} \kappa_{d} E_{d}=\sigma,
\end{gathered}
$$

with $\kappa_{b}$ being the background dielectric constant. Charge transport across the ferroelectric bulk can be described as

$$
\frac{d \sigma}{d t}=J\left(E_{f}\right)=\varepsilon_{0} \kappa^{*} \cdot \frac{d E_{f}}{d t}
$$

where $\kappa^{*}=\chi_{f}(\infty)+\kappa_{b}+\kappa_{d} h / d$. The initial condition for $E_{f}$ at $t=0$ obtained from Eq. (3) by using $\sigma=0$ and $P_{f}=P_{s}$ $+\varepsilon_{0} \chi_{f} E_{f}$ reads

$$
E_{f}(0)=-\frac{1}{\kappa^{*}}\left(\frac{P_{s}}{\varepsilon_{0}}-\kappa_{d} \frac{U}{d}\right) .
$$

For $J\left(E_{f}\right)$, a general exponential dependence can be assumed, encompassing several mechanisms of conduction in insulators: Pool-Frenkel mechanism $(\alpha=1, \beta=1 / 2)$, Schottky emission $(\alpha=0, \beta=1 / 2)$, tunneling transport $(\alpha=2, \beta=-1)$ 

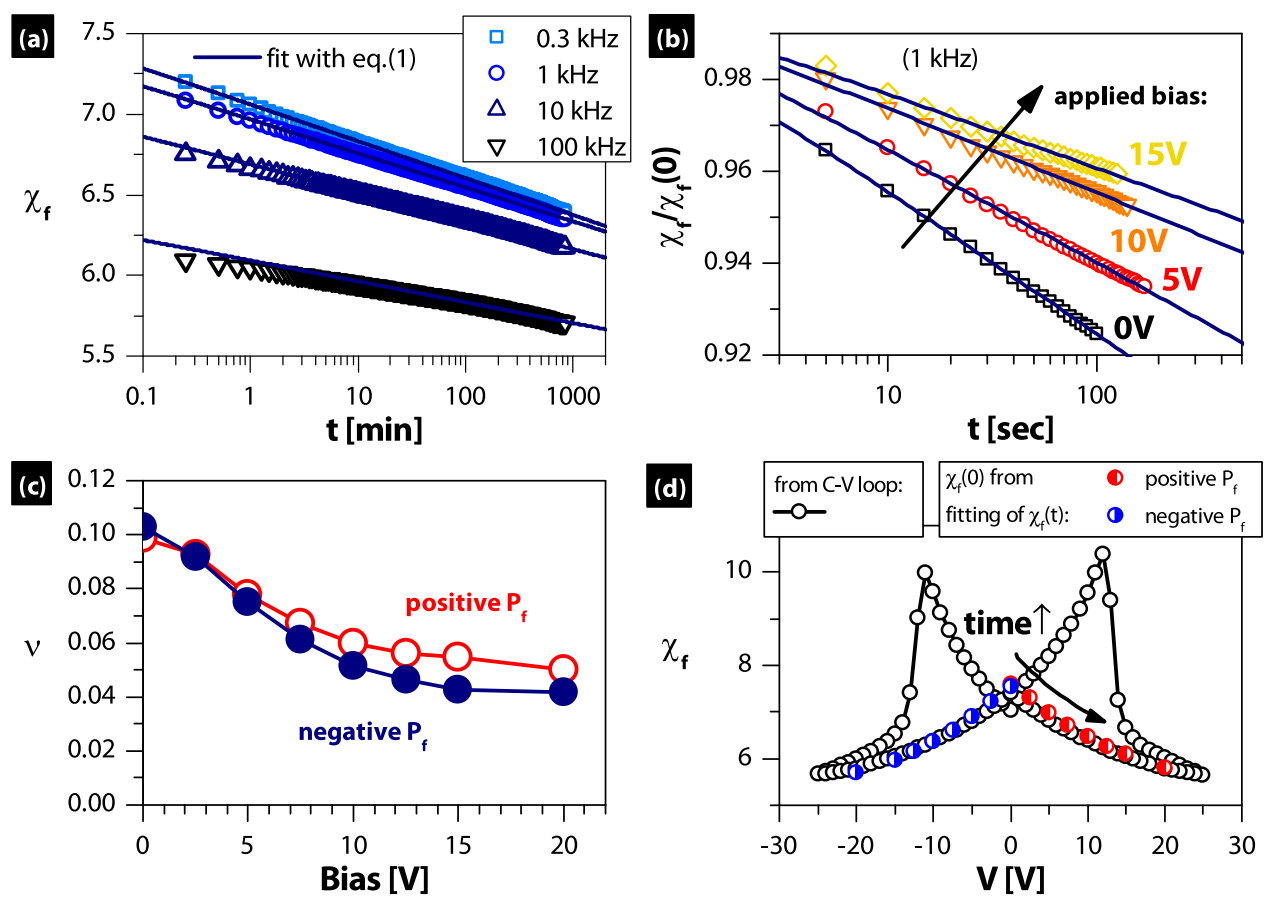

FIG. 3. (a) Dielectric response of $\mathrm{P}(\mathrm{VDF}-\mathrm{TrFE})$ as a function of time after poling $t$ for a range of frequencies. (b) Same measurement at $1 \mathrm{kHz}$ for several values of additional bias maintained after poling. (c) The degradation speed parameter $\nu$ extracted from fitting of experimental data as a function of applied bias. (d) $C$ - $V$ curve of $\mathrm{P}(\mathrm{DVF}-\mathrm{TrFE})$, the black arrow illustrates the process behind the $\chi_{f}(t)$ dependence as a descent along the curve due to compensation of $P_{f}$. The red and blue dots are the parameter values for $\chi_{f}(0)$ in Eq. (1), extracted from same data as in (b) and (c).

$$
J\left(E_{f}\right)=A \cdot\left(\frac{\left|E_{f}\right|}{E_{t h}}\right)^{\alpha} \cdot \exp \left(\operatorname{sign}(\beta) \cdot\left(\frac{\left|E_{f}\right|}{E_{t h}}\right)^{\beta}\right) \operatorname{sign}\left(E_{f}\right) .
$$

$A$ and $E_{t h}$ are mechanism-dependent constants. In the case where the charge trapped at the interface only weakly screens the bound charge of the polarization, solving Eqs. (4)-(6) for $E_{f}$ leads to

$$
\left|E_{f}\right|^{\beta}=\left|E_{f}(0)\right|^{\beta}-\frac{E_{t h}^{\beta}}{\operatorname{sign}(\beta)} \ln \left(1+\frac{t}{\tau_{0}}\right) .
$$

Thus, for the geometry shown in Fig. 2(c) where $E_{f}<0$, and for $\ln \left(1+t / \tau_{0}\right)$ not too large, the time dependence of $E_{f}$ is of the same kind as that of $\chi_{f}$ in Eq. (1)

$$
E_{f}=E_{f}(0)+G \cdot \ln \left(1+\frac{t}{\tau_{0}}\right)
$$

where $G=E_{t h}^{\beta}\left|E_{f}(0)\right|^{1-\beta} /|\beta|$.

Now, we turn to the evolution of $\chi_{f}$ in order to show that it closely follows $E_{f}$. In most general terms, the evolution of $\chi_{f}$ can be described via that of $P_{f}$. The process of timedependent compensation of $P_{f}$ can be illustrated as a descent along the $C-V$ curve (Fig. 3(c)). It should be noted that the results in Fig. 3(c) are measured on a composite sample incorporating non-ferroelectric regions; however, the $C-V$ curve is qualitatively the same for a pure ferroelectric layer. The decrease of $\chi_{f}$ is a result of compensation-induced decrease of $E_{f}$, increasing $P_{f}$. The system tends to $P_{f}=P_{s}$ and $\chi_{f}=\chi_{f}(\infty)$ for very large times. This is a fundamental process occurring independently of the type of ferroelectric material and its domain structure. A linearization of $\chi_{f}$ vs. $P_{f}$ dependence corresponds to a linear approximation of a small segment of the $\mathrm{C}-\mathrm{V}$ curve in Fig. 3(c) around $\mathrm{V}=0$ and yields the linear relation of $\chi_{f}$ vs. $E_{f}$

$$
\chi_{f}=\chi_{f}(0)-K \cdot\left(E_{f}-E_{f}(0)\right),
$$

where $K$ is a constant. This linear approximation implies the logarithmic time dependence of $\chi_{f}$ as described in Eq. (1). The dependence of factor $\nu$ in Eq. (1) on additional bias $(U)$ follows from the bias dependence of the factor $\mathrm{G}$ in Eq. (8). Combining Eqs. (1), (5), (8) and (9), one obtains

$$
v=K G=K \frac{E_{t h}^{\beta}}{|\beta|}\left(\frac{1}{\kappa^{*}}\left(\frac{P_{s}}{\varepsilon_{0}}-\kappa_{d} \frac{U}{d}\right)\right)^{1-\beta} .
$$

In our geometry application of the external electric field in the direction of polarization, vector implies $\mathrm{U}>0$ in Eq. (10). This corresponds to smaller $\nu$ and slower rate of the dielectric susceptibility decay with increasing electric field, as observed experimentally.

It is interesting to examine the possible reasons behind the emergence of $J\left(E_{f}\right)$ as the dominant polarization compensation mechanism in $\mathrm{P}(\mathrm{VDF}-\mathrm{TrFE})$. By assuming $U=0$ in Eqs. (2) and (3) (short circuit-condition), we can express the internal field in the ferroelectric as

$$
E_{f}=-\frac{1}{\varepsilon_{0}} \cdot \frac{P_{s}-\sigma}{\chi_{f}(\infty)+\kappa_{b}+\kappa_{d} h / d} .
$$

From this expression, it is clear that $E_{f}$ can only be big if: (I) $P_{s}$ is big, (II) dielectric response $\left(\chi_{f}(\infty)\right.$ and $\left.\kappa_{b}\right)$ of the ferroelectric is small, (III) $\kappa_{d} h / d$ is not too big. Conditions (I) and (II) are uniquely satisfied in $\mathrm{P}(\mathrm{VDF}-\mathrm{TrFE})$, where an appreciable remnant polarization of $\sim 9 \mu \mathrm{C} / \mathrm{cm}^{2}$ is combined with a low dielectric constant of $\sim 10$. Condition (III) implies that the thickness $d$ of the dielectric layer must be comparable with the ferroelectric layer $h$. This requirement can be satisfied in the multilayer-like material structure of a polymer ferroelectric. Thus, $\mathrm{P}(\mathrm{VDF}-\mathrm{TrFE})$ films represent a unique system that meets all conditions for the polarization screening driven by the depolarization field $E_{f}$. 
The leakage data collected on similar P(VDF-TrFE) structures ${ }^{13}$ suggest that the charge transport across the ferroelectric layer can be sufficient for the polarization screening. The reported current densities of $10^{-8}-10^{-7} \mathrm{~A} / \mathrm{cm}^{2}$ measured at the electric field of $10-100 \mathrm{kV} / \mathrm{cm}$ are sufficient for a significant degree of compensation that may be produced within the time scale of 1-1000 s. On the other hand, this analysis can be more complicated if the leakage originates from the defect-related filament conduction. Further transport studies in $\mathrm{P}(\mathrm{VDF}-\mathrm{TrFE})$ layers are required in order to clarify this point.

The picture of charge compensation through the ferroelectric bulk developed here is of practical importance in the context of polymer ferroelectric-based memories. Time dependence of capacitance is commonly used as a gauge of polarization stability in vertically asymmetric metal/ferroelectric/insulator/semiconductor (MFIS) structures (see, e.g., Refs. 14-17). In this study conducted in a symmetric metalferroelectric-metal (MFM) structure, we identified a mechanism, which should be intrinsic to P(VDF-TrFE) and not to a particular device structure. While not associated with polarization back-switching, ${ }^{18}$ it can superpose with such effect in the time dependence of the dielectric response.

The bulk mechanism of charge compensation presented in this study has important practical implications concerning the optimization of retention performance of the ferroelectric polymer field effect devices. Here, the approach differs fundamentally from the traditional perovskite ferroelectrics where a high retention performance is achieved by using best insulators as buffer layers. ${ }^{19}$ In particular, we show that in P(VDF-TrFE) the polarization screening occurs due to the charge transport through the bulk. Hence instead of improving the insulating properties of the interface-adjacent buffer layers, the insulating properties of the ferroelectric itself have to be enhanced. From practical prospective, the promising approaches to control the polarization screening in $\mathrm{P}(\mathrm{VDF}-\mathrm{TrFE})$ layers may include control of crystallinity and/or chemical composition of the polymer by adding dopants or additives.
The research has received funding from the Swiss National Science Foundation Grant no. 132724 and the European Research Council under the European Union's Seventh Framework Programme (FP7/2007-2013)/ERC Grant Agreement No. 268058.

${ }^{1}$ S. Horiuchi and Y. Tokura, Nature Mater. 7, 357-366 (2008).

${ }^{2}$ M. Poulsen and S. Ducharme, IEEE Trans. Dielectr. Electr. Insul. 17, 1028-1035 (2010).

${ }^{3}$ T. Furukawa, Y. Takahashi, and T. Nakajima, Curr. Appl. Phys. 10, e62-e67 (2010).

${ }^{4}$ R. C. G. Naber, C. Tanase, P. W. M. Blom, G. H. Gelinck, A. W. Marsman, F. J. Touwslager, S. Setayesh, and D. M. de Leeuw, Nature Mater. 4, 243-248 (2005).

${ }^{5}$ K. Asadi, D. M. de Leeuw, B. de Boer, and P. W. M. Blom, Nature Mater. 7, 547-50 (2008).

${ }^{6}$ R. C. G. Naber, K. Asadi, P. W. M. Blom, D. M. de Leeuw, and B. de Boer, Adv. Mater. 22, 933-45 (2010).

${ }^{7}$ Y. Park, I. Bae, S. Kang, J. Chang, and C. Park, IEEE Trans. Dielectr. Electric. Insul. 17, 1135-1163 (2010).

${ }^{8}$ I. Stolichnov, S. W. E. Riester, H. J. Trodahl, N. Setter, A. W. Rushforth, K. W. Edmonds, R. P. Campion, C. T. Foxon, B. L. Gallagher, and T. Jungwirth, Nature Mater. 7, 464-467 (2008).

${ }^{9}$ Y. Yuan, T. J. Reece, P. Sharma, S. Poddar, S. Ducharme, A. Gruverman, Y. Yang, and J. Huang, Nature Mater. 10, 296-302 (2011).

${ }^{10}$ M. Grossmann, O. Lohse, D. Bolten, U. Boettger, T. Schneller, and R. Waser, J. Appl. Phys. 92, 2680 (2002).

${ }^{11}$ A. K. Tagantsev, I. Stolichnov, N. Setter, and J. S. Cross, J. Appl. Phys. 96, $6616(2004)$

${ }^{12}$ M. Grossmann, O. Lohse, D. Bolten, U. Boettger, T. Schneller, and R. Waser, Appl. Phys. Lett. 80, 1427 (2002).

${ }^{13}$ S. Fujisaki, H. Ishiwara, and Y. Fujisaki, Appl. Phys. Express 1, 081801 (2008).

${ }^{14}$ A. Gerber, H. Kohlstedt, M. Fitsilis, R. Waser, T. J. Reece, S. Ducharme, and E. Rije, J. Appl. Phys. 100, 024110 (2006).

${ }^{15}$ T. N. Ng, B. Russo, and A. C. Arias, J. Appl. Phys. 106, 094504 (2009).

${ }^{16}$ T. J. Reece, A. Gerber, H. Kohlstedt, and S. Ducharme, J. Appl. Phys. 108, 024109 (2010).

${ }^{17}$ K. Müller, K. Henkel, D. Mandal, B. Seime, I. Paloumpa, and D. Schmeißer, Phys. Status Solidi A 208, 330-342 (2011).

${ }^{18}$ R. Gysel, I. Stolichnov, A. K. Tagantsev, S. W. E. Riester, N. Setter, G. A. Salvatore, D. Bouvet, and A. M. Ionescu, Appl. Phys. Lett. 94, 263507 (2009).

${ }^{19}$ K. Aizawa, B.-E. Park, Y. Kawashima, K. Takahashi, and H. Ishiwara, Appl. Phys. Lett. 85, 3199 (2004). 